IU/NTC 97-12

\title{
Parity Violating Elastic Electron Scattering and Coulomb Distortions
}

\author{
C. J. Horowitz* \\ Nuclear Theory Center \\ 2401 Milo B. Sampson Lane \\ Bloomington, Indiana 47405 USA
}

(May 12, 2018)

\begin{abstract}
Parity violating elastic electron-nucleus scattering provides an accurate and model independent measurement of neutron densities, because the $Z^{0}$ couples primarily to neutrons. Coulomb distortion corrections to the parity violating asymmetry $A_{l}$ are calculated exactly using a relativistic optical model. Distortions significantly reduce $A_{l}$ in a heavy nucleus. However even with distortions, an experiment to measure the neutron radius is feasible. This will aid the interpretation of future atomic parity violation measurements and provide fundamental nuclear structure information. Coulomb distortions and small differences between neutron and proton radii could be important for a standard model test on ${ }^{4} \mathrm{He},{ }^{12} \mathrm{C}$ or ${ }^{16} \mathrm{O}$.
\end{abstract}

$24.80+\mathrm{y}, 25.30 . \mathrm{Bf}, 21.10 . \mathrm{Gv}$

Typeset using REVTEX

*email: charlie@iucf.indiana.edu 


\section{INTRODUCTION}

Parity violating electron-nucleus scattering is important for several reasons. First, it provides a test of the standard model at low energies. Indeed, an early experiment on ${ }^{12} \mathrm{C}$ was performed [1] (with somewhat limited accuracy). Second, it is sensitive to strange quarks in the nucleon or nucleus. Proposed Jefferson Laboratory experiments hope to extract strange quark contributions to the electric form factor of the nucleon from elastic scattering on ${ }^{4} \mathrm{He}[2]$. Finally, parity violation provides a unique and very clean way to study neutron densities and isospin violation in nuclei [3]. This is because the $Z^{0}$ couples predominantly to neutrons. [Note, the $Z^{0}$ proton coupling depends on the small factor $1-4 \sin ^{2} \Theta_{W}$.]

An accurate measurement of neutron distributions in a heavy nucleus would provide fundamental nuclear structure information. It will constrain isovector terms in the nuclear matter energy functional such as the surface symmetry energy. This could be important in astrophysics when one extrapolates to unstable very asymmetric nuclei. Furthermore, a measurement of neutron radii will significantly aid the interpretation of future atomic parity violation measurements. The present $C s$ experiment is accurate to 0.3 percent [4]. Pushing the accuracy of atomic experiments is important as a test of the standard model and as a search for new physics. However, a 0.1 percent measurement in a heavy atom will require knowing the neutron radius to of order one percent [5] (to keep uncertainties in the neutron density from interfering with a standard model test).

The charge density is known from elastic electron scattering. Thus, to determine the neutron radius to one percent requires knowing the difference between neutron and proton radii to about 25 percent. This accuracy is probably beyond that of present nuclear theory (we comment on this below). Furthermore, neutron radii determinations from hadronic probes suffer from large systematic errors. Therefore, a measurement of the parity violating asymmetry for elastic electron scattering should provide crucial information for atomic parity experiments.

Atomic experiments also depend on atomic theory (the overlap of electronic wave function with the nucleus) which at present is only good to about one percent in Cs [6]. However, this accuracy may improve in the future. If this is not the case, atomic experiments may shift to measuring ratios of parity violation in different isotopes because many of the atomic uncertainties cancel. Isotope ratios place much more stringent requirements on knowledge of the neutron radius and how this changes among isotopes. This knowledge is beyond present nuclear theory. However, an accurate measurement of the neutron radius with electron scattering on a single nucleus should still provide an important first step towards calibrating a theory of neutron radii differences.

Electron scattering from a heavy nucleus is modified substantially by coulomb distortions. These effects are of order $Z \alpha$ (where $Z$ is the nuclear charge) and will modify the parity violating asymmetry. However, there are no previously published calculations. In the present paper we accurately calculate coulomb distortion effects with a relativistic optical model. The Dirac equation is numerically solved for an electron moving in vector and axial vector potentials. Our formalism is presented in section II along with checks of the numerics.

Elastic parity violating asymmetries from several nuclei are shown in section III. Results are also shown for a variety of electron energies. We conclude in section IV that coulomb distortions significantly modify the asymmetry. However these are accurately calculated. 
Even with distortions, the asymmetry is very sensitive to neutron densities and an experiment to measure the neutron radius in a heavy nucleus is feasible. We also conclude that coulomb distortions are important for a one percent standard model test in ${ }^{12} \mathrm{C}$ or ${ }^{16} \mathrm{O}$ and that such a test may be sensitive to very small differences in proton and neutron radii.

\section{FORMALISM}

In this section we describe our relativistic optical model formalism and discuss a variety of checks on our numerical results. The electron wave function $\Psi$ (for scattering from a spin zero nucleus) is assumed to satisfy a Dirac equation,

$$
\left[\alpha \cdot \mathbf{p}+\beta m_{e}+\hat{V}(r)\right] \Psi=E \Psi .
$$

Here $E$ is the center of mass energy and we neglect other center of mass corrections. The total potential $\hat{V}(r)$ has vector $V(r)$ and axial vector $A(r)$ components,

$$
\hat{V}(r)=V(r)+\gamma_{5} A(r) .
$$

The conventional coulomb potential is $V$ while weak neutral currents give rise to $A$ which is of order the Fermi constant $G_{F}$,

$$
A(r)=\frac{G_{F}}{2^{3 / 2}} \rho_{W}(r)
$$

The weak charge density $\rho_{W}$ is closely related to (minus) the neutron density (see below) and is normalized, for neutron number $N$ and proton number $Z$,

$$
\int d^{3} r \rho_{W}(r)=-N+\left(1-4 \sin ^{2} \Theta_{W}\right) Z
$$

Equation (1) includes terms of all orders in $Z \alpha$. This is important because $Z \alpha$ is large for a heavy nucleus. Equation (1) neglects radiative corrections, which are higher order in $\alpha$, and dispersion corrections where the intermediate nucleus is in an excited state.

In the limit of vanishing electron mass, it is a simple matter to include the effects of the axial potential $A(r)$. One writes the Dirac equation for helicity states with $\Psi_{ \pm}=\frac{1}{2}\left(1 \pm \gamma_{5}\right) \Psi$,

$$
\left[\alpha \cdot \mathbf{p}+V_{ \pm}(r)\right] \Psi_{ \pm}=E \Psi_{ \pm}
$$

and

$$
V_{ \pm}(r)=V(r) \pm A(r) .
$$

Thus, the positive helicity state scatters from a potential $V+A$ while the negative helicity state scatters from $V-A$. To calculate the parity violating asymmetry $A_{l}$ one simply calculates the scattering amplitudes for $V+A$ and $V-A$ and subtracts,

$$
A_{l}=\frac{d \sigma_{+} / d \Omega-d \sigma_{-} / d \Omega}{d \sigma_{+} / d \Omega+d \sigma_{-} / d \Omega} .
$$


We have written a new relativistic optical code ELASTIC which numerically solves the partial wave Dirac equation and sums up phase shifts to calculate the scattering amplitude. From the amplitude it calculates the following observables: the unpolarized cross section, the parity conserving analyzing power $A_{y}$ (for an initial electron spin normal to the reaction plane), the parity violating asymmetry $A_{l}$ and the spin rotation parameter $Q$. This is related to the angle through which the electron's spin is rotated when it scatters from the nucleus [7]. The normal analyzing power $A_{y}$ is of order $m_{e} / E$ and vanishes in Born approximation. It is very small, comparable to $A_{l}$ ! We will discuss $A_{y}$ in a future paper.

The numerical details of the code will also be presented in a later paper. Here we describe some of the checks which give us confidence in our results. First the code must reproduce known cross sections. For example, elastic cross sections from ${ }^{208} \mathrm{~Pb}$ at $502 \mathrm{MeV}$ are reproduced out to $3.7 \mathrm{Fm}^{-1}$ (the extent of the data [8]). Near $3.7 \mathrm{Fm}^{-1}$ the error is of order a percent. The cross section at these large angles is reduced by many orders of magnitude. This requires that the scattering amplitude be calculated very accurately: indeed, more accurately then needed for the forward angle $A_{l}$ (see below).

The code has been checked against plane wave results. We multiplied both $V$ and $A$ in Eqs. $(1,2)$ by a small factor, say 0.01 . Then the full code was run summing over many partial waves. Finally the resulting cross section was divided by $0.01^{2}$ and both the cross section and $A_{l}$ were compared to know plane wave results. This check was performed both for $\rho_{W}$ proportional to the charge density (where $A_{l}$ is linear in $q^{2}$ ) and for different neutron and proton densities. The numerical agreement is very good (better then 0.1 percent for $A_{l}$ ) except right in the diffraction minima. In the minima, the exact result should be different from plane wave results even for a system with the small charge of $0.01 Z$. This plane wave test is actually more demanding then the full calculation because some numerical errors are magnified in comparison to the small interaction.

The spin rotation parameter $Q$ should be just,

$$
Q=\sin (\Theta),
$$

with $\Theta$ the electron scattering angle, up to small corrections of order $m_{e} / E$. This is a nontrivial check since we must sum up over all partial waves to calculate $Q$. Equation (8) is reproduced by our code except at very large momentum transfers (beyond $4 \mathrm{Fm}^{-1}$ for ${ }^{208} \mathrm{~Pb}$ where the cross section is also inaccurate).

Finally, the only new feature of the $A_{l}$ calculation is a subtraction of the positive and negative helicity amplitudes. In practice this is not a problem because the amplitude must be calculated to much better accuracy then a part in $10^{5}$ (a typical size for $A_{l}$ ) in order to reproduce the large angle cross section. Furthermore, many errors cancel in the subtraction. Nevertheless, this can be tested by multiplying just $A$ in Eq. (3) by 0.1 (keeping $V$ unchanged) and running the full code. The resulting $A_{l}$ is scaled up by a factor of ten and seen to agree well with earlier results. This procedure makes the subtraction ten times more sensitive and verifies its accuracy. Note, the code calculates observables to all orders in both $V$ and $A$. In practice, $A$ is small so $A_{l}$ is linear in $A$. Therefore, the code can be run with almost any value of $G_{F}$ in Eq. (3) and the resulting output $A_{l}$ scaled appropriately.

Taken together, these four tests check almost all areas of the calculation and give us confidence in our results. In practice the calculation is no harder then older work for the unpolarized cross section. Indeed, in a helicity basis only very small modifications are needed 
to include a parity violating potential.

\section{RESULTS}

In this section we present results of the code ELASTIC. We first assume the weak density $\rho_{W}(r)$ has the same spatial distribution as the charge density $\rho(r)$. This allows one to see the effects of only coulomb distortions. Then we show results for different weak and electromagnetic densities. To start, we use a simple three parameter Fermi charge density for ${ }^{208} \mathrm{~Pb}$ from ref. [9], see Table I. This fits all but the back angle electron scattering data. The weak density is assumed to be proportional to the charge density,

$$
\rho_{W}(r)=-\left[\frac{N}{Z}+4 \sin ^{2} \Theta_{W}-1\right] \rho(r) .
$$

This satisfies the normalization condition of Eq. (4). For simplicity in notation, we refer to the weak density given by Eq. (9) as being equal to the charge density.

Figure 1 shows the asymmetry $A_{l}$ for ${ }^{208} \mathrm{~Pb}$ versus momentum transfer $q$ both in a plane wave impulse approximation where,

$$
A_{l}=\left[\frac{G_{F} q^{2}}{4 \pi \alpha 2^{1 / 2}}\right]\left[\frac{N}{Z}+4 \sin ^{2} \Theta_{W}-1\right]
$$

and then including full distortions at electron energies from 502 to $3000 \mathrm{MeV}$. Coulomb distortions are seen to reduce $A_{l}$ substantially, especially in the diffraction minima. As the energy increases, the effects of coulomb distortions do not decrease (very much) instead there is a slight shift in the position of the diffraction minima to higher momentum transfers. We conclude from Fig. 1 that coulomb distortions must be included for parity violation in a heavy nucleus.

Figure 2 shows $A_{l}$ for ${ }^{12} \mathrm{C}$ at $200 \mathrm{MeV}$. This is the energy of the original BATES experiment[1]. This figure uses a relativistic mean field model [10](MFT) for the charge density. For the MFT we approximate the weak density as,

$$
\rho_{W}(r)=\int d^{3} r^{\prime} G_{E}\left(\left|\mathbf{r}-\mathbf{r}^{\prime}\right|\right)\left[-\rho_{n}\left(r^{\prime}\right)+\left(1-4 \sin ^{2} \Theta_{W}\right) \rho_{p}\left(r^{\prime}\right)\right] .
$$

Here $\rho_{n}$ and $\rho_{p}$ are point neutron and proton densities and the electric form factor of the proton is approximated $G_{E}(r) \approx \frac{\Lambda^{3}}{8 \pi} e^{-\Lambda r}$ with $\Lambda=4.27 \mathrm{Fm}^{-1}$. This neglects strange quark contributions, the neutron electric form factor and meson exchange currents. It also assumes good isospin for the nucleon. For simplicity, all calculations in this paper use $\sin ^{2} \Theta_{W}=0.23$ for the Weinberg angle.

The dotted curve in Fig. 2 assumes Eq. (9) while the dashed curve uses the MFT weak density. Both of these are plane wave calculations. Finally, the solid curve uses the MFT weak density and includes coulomb distortions. In the MFT the protons have a slightly larger radius then the neutrons because of coulomb repulsion. This small change in radius can lead to a large change in $A_{l}$ at back angles. At the $30 \mathrm{deg}$. angle of the BATES experiment the MFT plane wave calculation is about $1 \%$ above the equal density plane 
wave result. Coulomb distortions increase $A_{l}$ by another two \%. Thus the full calculation is about $3 \%$ above the original prediction. This change is smaller then the BATES error. However, it is large compared to a possible one \% standard model test.

We conclude that coulomb distortions must be included in a one $\%$ standard model test on ${ }^{12} \mathrm{C}$ or ${ }^{16} \mathrm{O}$ (indeed Fig. 3 shows similar results for ${ }^{16} \mathrm{O}$ ). However, we have calculated coulomb distortions accurately so they should pose no problems for the interpretation of the experiment. We also see that isospin violation (small differences between proton and neutron densities) is significant especially at back angles. This correction involves some nuclear structure uncertainties. Thus isospin violation may limit a standard model test to small momentum transfers.

Figure 4 shows $A_{l}$ for ${ }^{4} \mathrm{He}$ at $850 \mathrm{MeV}$. We assume a 3 parameter Fermi charge density, see Table I. For this light target, coulomb distortions are only important in the diffraction minima. The solid curve is for a neutron density arbitrarily one $\%$ smaller then the proton density. This change in $r_{n}$ is somewhat bigger then theoretical estimates. However, there is great sensitivity to small changes in the neutron density. An accurate microscopic calculation of $r_{n}-r_{p}$ using Greens Function Monte Carlo or other methods is very important.

We note that the solid curve crosses the dashed curve just beyond 50 degrees. This is near the second maximum in the form factor and corresponds to the kinematics of a planned experiment [2]. At this momentum transfer, $q$, the derivative of the cross section with $q$ goes to zero which reduces some systematic errors (such as those from helicity correlated changes in $q$ ). One can think of the derivative as being with respect to the dimension-less quantity $q r$ with $r$ the nuclear radius. Thus the derivative of the cross section with respect to $r$ also vanishes at the same point. This implies that the cross section and asymmetry will be insensitive to small changes in $r$ or $r_{n}-r_{p}$. This minimizes the sensitivity to isospin violation. However the sensitivity is large at other momentum transfers.

Results for $A_{l}$ in ${ }^{208} \mathrm{~Pb}$ at $850 \mathrm{MeV}$ are shown in Fig. 5 at forward angles and in Fig. 6 at backward angles. [Note, all of the curves in these and remaining figures include coulomb distortions.] The dotted curve assumes a three parameter Fermi charge density and equal weak density. The solid curve uses relativistic mean field (MFT) [10] charge and weak densities. The large difference between these curves indicates a strong sensitivity to the neutron radius or $r_{n}-r_{p}$. Finally, the dashed curve assumes the weak density is a scaled (stretched) version of the (three parameter Fermi) charge density,

$$
\rho_{W}(r)=-\left[\frac{N}{Z}+4 \sin ^{2} \Theta_{W}-1\right] \lambda^{3} \rho(\lambda r) .
$$

The scale parameter $\lambda=.9502$ is chosen to reproduce the MFT $r_{n}-r_{p}$. These various densities are shown in Fig. 7. Root mean square radii are collected in table II. The good agreement between the dashed and solid curves in Fig. 5 indicates that a forward angle measurement is primarily sensitive to the neutron radius and not to shell structure in the density (see Fig. 7). The scaled three parameter Fermi density is very different from the MFT at small $r$.

The nucleus ${ }^{138} \mathrm{Ba}$ provides a meeting ground between nuclear and atomic physics. There is interest in an atomic parity violation experiment on the $\mathrm{Ba}$ ion. At the same time, ${ }^{138} \mathrm{Ba}$ has a relatively simple nuclear structure and a large gap of about $1.5 \mathrm{MeV}$ to the first excited state. [Unfortunately Cs isotopes have relatively complicated nuclear structure and low first 
excited states.] Thus Ba may be a good place to measure both the neutron radius (with electron scattering) and atomic parity violation. Figure 8 shows MFT weak and charge densities for ${ }^{138} \mathrm{Ba}$ and Fig. 9 presents parity violating asymmetries. The sensitivity to the neutron radius is large, comparable to $\mathrm{Pb}$.

Alternatively, one may be able to accurately calibrate nuclear theory with a measurement of $r_{n}-r_{p}$ in ${ }^{208} \mathrm{~Pb}$ and then use theory to interpolate to other nuclei of interest in atomic physics. In a latter paper we will discuss both the absolute errors in nuclear theory and the relative errors in going from one nucleus to another. It should be possible to achieve the needed one percent relative error. Thus one may be able to understand bulk neutron radii throughout the periodic table with only a single measurement.

From a nuclear structure point of view alone, an obvious choice is ${ }^{208} \mathrm{~Pb}$ since this is such a good doubly closed shell nucleus with a simple structure, a high first excited state, a large cross section and a large neutron excess. We also show in Figs. 10 and 11 predictions for ${ }^{48} \mathrm{Ca}$ since this nucleus has a large fractional neutron excess. Again, there is a large sensitivity to the neutron radius. However, the cross section for ${ }^{48} \mathrm{Ca}$ is smaller then for ${ }^{208} \mathrm{~Pb}$.

We now discuss the optimal kinematics for an experiment on ${ }^{208} \mathrm{~Pb}$. The figure of merit,

$$
F=A_{l}^{2} d \sigma / d \Omega,
$$

is shown in Fig. 12 for $850 \mathrm{MeV}$. This is strongly forward peaked since the cross section falls rapidly with angle. Thus experiments may only be feasible at forward angles. Figure 5 suggests one is sensitive to the neutron density at scattering angles near 6 and 12 degrees in the Lab. This is made quantitative in Fig. 13 where we plot the logarithmic derivative of the asymmetry with respect to the scale factor of the neutron density, $\lambda$ of Eq. 11 (equivalently with respect to the neutron radius),

$$
\frac{d \log A_{l}}{d \log \lambda}=\frac{\lambda}{A_{l}}\left(\frac{d A_{l}}{d \lambda}\right)
$$

Note, this is evaluated at $\lambda=.9502$. The logarithmic derivative peaks around 3.2 near 7 degrees and around 10 near 14 degrees. A value of 3.2 means that a 3.2 percent measurement of $A_{l}$ could determine the neutron radius to one percent (if other uncertainties are small).

The product of the figure of merit and the logarithmic derivative is also shown in Fig. 12. This is large where $A_{l}$ can be accurately measured and is sensitive to the neutron radius. The first maximum in this product (near 4 degrees) is about 20 times the second maximum (near 12 degrees). Figure 12 is for a fixed beam energy of $850 \mathrm{MeV}$. Results can be approximately scaled to other energies, $E$, by multiplying the cross section (at fixed momentum transfer) by $(E / 850 \mathrm{MeV})^{2}$.

For example, an experiment at a fixed laboratory angle of six degrees is illustrated in Fig. 14. Note, this is an approximate figure since it is based on distortion calculations at $850 \mathrm{MeV}$ scaled to other energies. However, it should provide a good first orientation. A measurement at six degrees is possible in Hall A at Jefferson Lab. with a septum magnet. Figure 14 has local maxima near 730, 1720 and $2600 \mathrm{MeV}$. The product of figure of merit times log. derivative is a factor of 3.8 (15) lower at 1720 (2600) MeV then at $730 \mathrm{MeV}$.

A measurement near $730 \mathrm{MeV}$ is insensitive to possible uncertainties in the surface thickness (of the neutron density). However, the surface thickness may be well known from 
theory where it is constrained by the surface energy. A measurement near $1720 \mathrm{MeV}$ is more sensitive to the neutron radius (see Fig. 13) so it may be less sensitive to other corrections or errors. Therefore it would be very useful to measure both points. However, most of the information on the neutron radius can be extracted from a single measurement. If pushed for time, it is most important to make a single accurate measurement (then two less accurate ones).

\section{CONCLUSIONS}

In this paper we have calculated parity violating asymmetries for elastic electron scattering including coulomb distortions. We solve a relativistic optical model for electron scattering in vector and axial-vector potentials. A series of plane wave and cross section checks give us confidence in the numerical results.

Our most important conclusion is that a parity violation experiment to measure the neutron density in a heavy nucleus is feasible. Possible targets include ${ }^{208} \mathrm{~Pb}$ because of its simple structure, good closed shells, and large neutron excess or ${ }^{138} \mathrm{Ba}$ because of the overlap of atomic physics interest and a relatively simple nuclear structure. It is straight forward to optimize the kinematics of such an experiment. However, one must include the large effects of coulomb distortions. One possibility for ${ }^{208} \mathrm{~Pb}$ is to measure around six degrees and an energy near 750 and or $1700 \mathrm{MeV}$. We would be happy to provide more detailed calculations upon request.

Future atomic parity experiments will require accurate knowledge of the neutron radius. In a later paper, we explore how a single good electron scattering measurement, coupled with the many constraints of nuclear theory, should be enough to predict the neutron radius to one percent for all closed shell nuclei. Note, determining small differences between isotopes is clearly more demanding. However, an understanding of bulk neutron radii is still an important first step towards a theory of isotope differences.

A measurement of the neutron radius will also provide fundamental nuclear structure information. It would be the first accurate and model independent measurement of the size of large hadronic systems. Note, the size does not follow directly from the charge radius because of the neutron skin. The measurement will provide important constraints on the isospin dependence of the nuclear matter energy functional and should constrain parameters such as the surface symmetry energy and or the isovector incompressibility.

We have found that coulomb distortions are also important for a one percent standard model test in ${ }^{12} \mathrm{C}$ or ${ }^{16} \mathrm{O}$. However, we have calculated distortions accurately so they should not pose a problem in the interpretation of an experiment. Small differences between proton and neutron radii are also important for elastic experiments involving ${ }^{4} \mathrm{He},{ }^{12} \mathrm{C}$ and ${ }^{16} \mathrm{O}$. Microscopic calculations of the difference between neutron and proton radii in ${ }^{4} \mathrm{He}$ or ${ }^{16} \mathrm{O}$ would be very useful.

\section{ACKNOWLEDGMENTS}

I thank Tim Cooper for a great deal of very valuable help on the numerics. Bill Donnelly, Steve Pollock and Mike Ramsey-Musolf are thanked for useful physics discussions. I thank 
Bunny Clark for electron scattering data and Dick Furnstahl and Horst Muller for neutron densities. Supported in part by DOE grant number DE-FG02-87ER-40365. 


\section{REFERENCES}

[1] Souder P.A., et al., Phys. Rev. Lett. 65 (1990) 694.

[2] CEBAF Proposal No. PR-91-004, E.J. Beise, spokesperson (unpublished). See also for example, Musolf M.J., Schiavilla R. and Donnelly T.W. Phys. Rev. C50 (1994) 2173.

[3] Donnelly T.W., Dubach J. and Sick Ingo, Nuc. Phys. A503 (1989)589.

[4] Wood C.S., Bennett S.C., Cho D., Masterson B.P., Roberts J.L., Tanner C.E. and C.E. Wienmann, Science 275 (1997) 1759.

[5] Pollock S.J., Fortson E.N. and Wilets L., Phys. Rev. C46 (1992) 2587. James J. and Sandars P.G.H., "A Parametric Approach to Nuclear Size and Shape in Atomic Parity Nonconservation", Univ. of Oxford Preprint to be published.

[6] Blundell S.A., Sapirstein J. and Johnson W.R., Phys. Rev. D45 (1992) 1602. Dzuba V., Flambaum V. and Sushkov O., Phys. Lett. 141A (1989)147.

[7] See for example Rahbar A. et al, Phys. Rev. Lett. 47 (1981) 1811.

[8] Frois B. et al, Phys. Rev. Lett. 38 (1977)152.

[9] Atomic Data and Nuclear Data Tables, Vol. 14 (1974) 479 and B.C. Clark private comunication.

[10] Horowitz C.J. and Serot B.D., Nuc. Phys.A368(1981)503. 


\section{TABLES}

TABLE I. Three parameter Fermi Densities [9] $\rho=\rho_{o}\left[1+w(r / R)^{2}\right] /[1+\exp ((r-R) / a)]$

\begin{tabular}{cccc}
\hline \hline Nucleus & $\mathrm{R}$ & $\mathrm{a}$ & $\mathrm{W}$ \\
\hline & $F m$ & $F m$ & \\
${ }^{4} \mathrm{He}$ & 1.008 & 0.327 & 0.445 \\
${ }^{208} \mathrm{~Pb}$ & 6.4 & 0.54 & 0.32 \\
\hline \hline
\end{tabular}

TABLE II. Root mean square radii for densities used

\begin{tabular}{cccc}
\hline \hline Nucleus & Density & Charge (Fm) & Weak (Fm) \\
\hline${ }^{4} \mathrm{He}$ & $3 \mathrm{p}$ 円 & 1.717 & 1.717 \\
${ }^{12} \mathrm{C}$ & $\mathrm{MFT}$ & 2.504 & 2.477 \\
${ }^{16} \mathrm{O}$ & $\mathrm{MFT}$ & 2.753 & 2.720 \\
${ }^{48} \mathrm{Ca}$ & $\mathrm{MFT}$ & 3.419 & 3.667 \\
${ }^{138} \mathrm{Ba}$ & $\mathrm{MFT}$ & 4.797 & 5.038 \\
${ }^{208} \mathrm{~Pb}$ & $3 \mathrm{p}$ & 5.490 & 5.490 \\
& $3 \mathrm{p}$ & & 5.778 \\
& $\mathrm{MFT}$ & $5.9502)$ & 5.756 \\
\hline \hline
\end{tabular}

${ }^{1}$ Three parameter Fermi function, see Table 1. MFT $=$ relativistic mean field theory densities from reference [10].

${ }^{2}$ Densities for ${ }^{48} \mathrm{Ca}$ include a small correction from a nonzero neutron electric form factor $G_{E}^{n}$. 


\section{FIGURES}

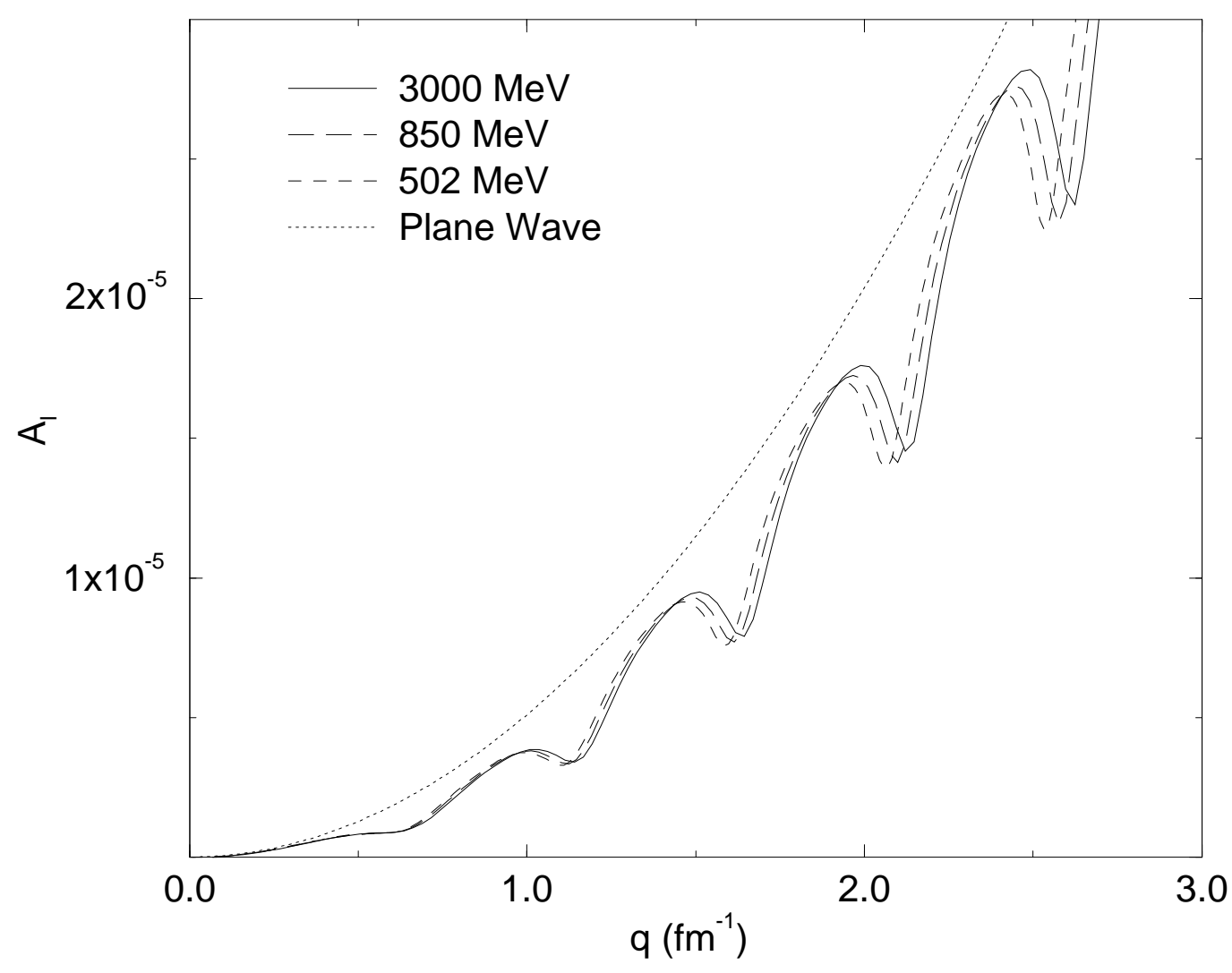

FIG. 1. Parity violating asymmetry $A_{l}$ for elastic scattering from ${ }^{208} \mathrm{~Pb}$ vs. momentum transfer $q$ assuming "equal" weak and charge densities (which are taken to be three parameter Fermi functions) see Eq. (9). The dotted curve is a plane wave approximation while full distorted wave results at $502 \mathrm{MeV}$ are short dashed, $850 \mathrm{MeV}$ long dashed and $3000 \mathrm{MeV}$ solid curves. 


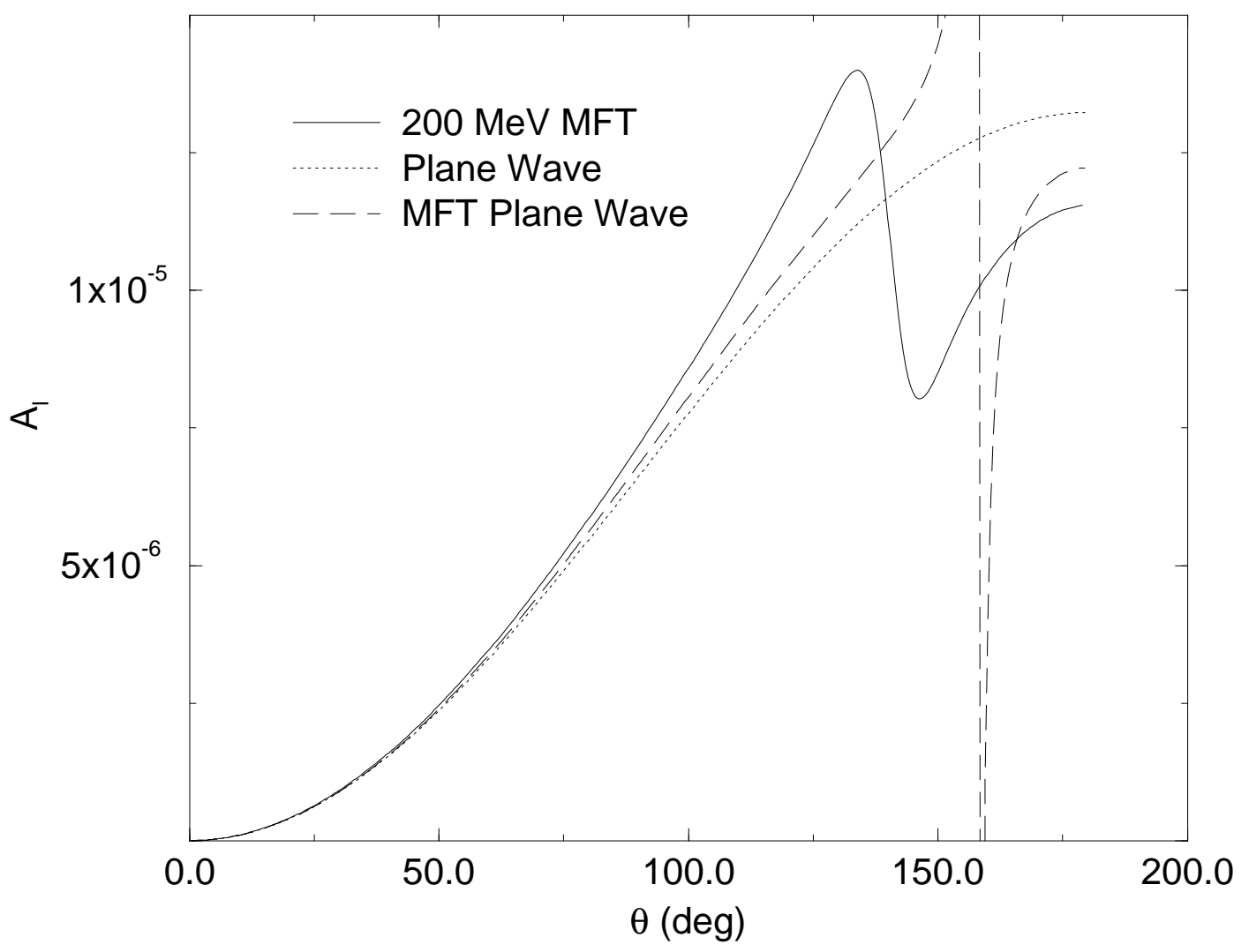

FIG. 2. Parity violating asymmetry $A_{l}$ for elastic scattering from ${ }^{12} \mathrm{C}$ at $200 \mathrm{MeV}$ vs. scattering angle $\theta$. Plane wave results using relativistic mean field densities (which are slightly different for neutrons and protons) are the dashed curve while the dotted curve is a plane wave calculation assuming equal neutron and proton densities. Finally, the solid curve is a full distorted wave calculation based on rel. mean field densities. 


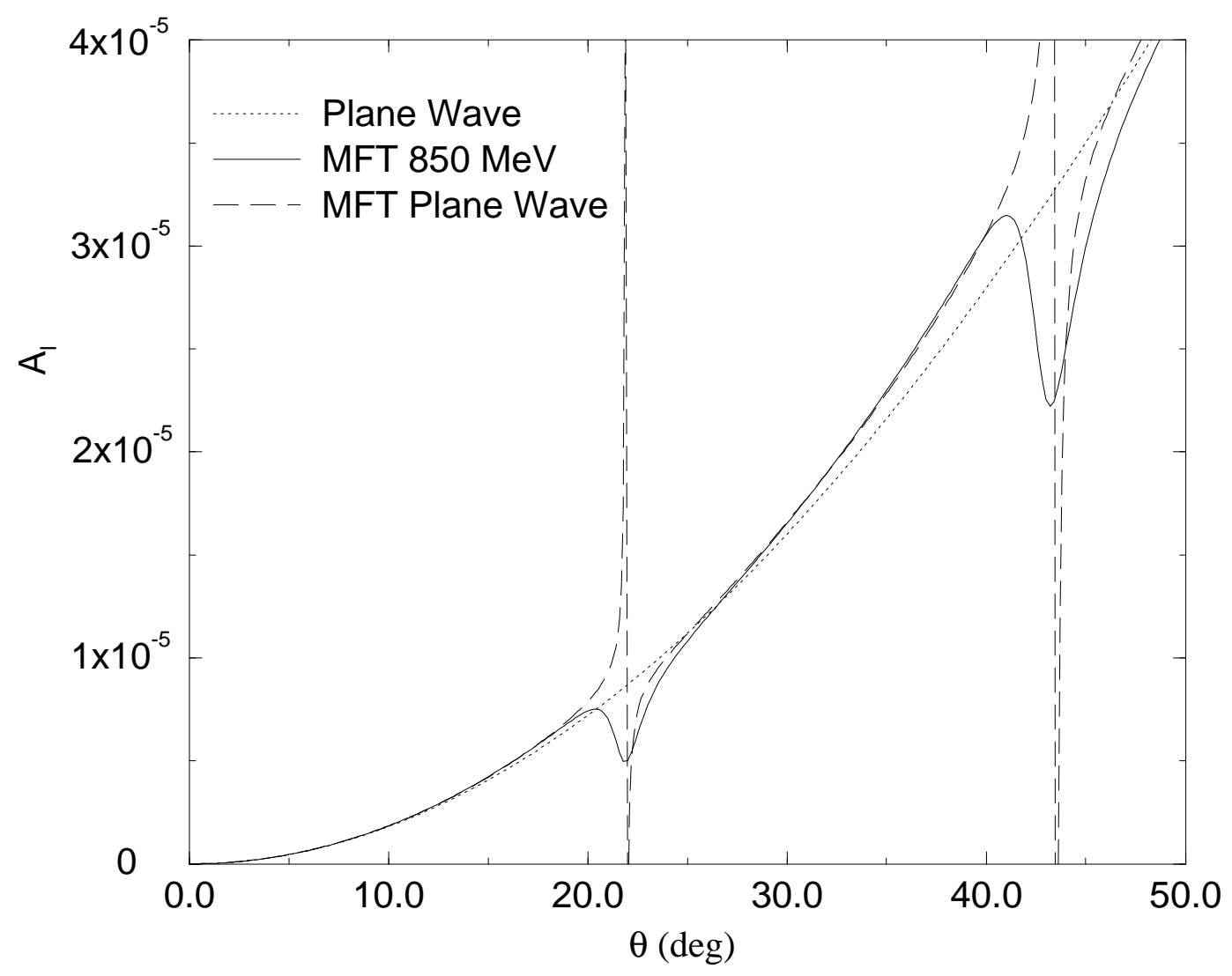

FIG. 3. Parity violating asymmetry $A_{l}$ for elastic scattering from ${ }^{16} \mathrm{O}$ at $850 \mathrm{MeV}$ vs. scattering angle $\theta$. Plane wave results using relativistic mean field densities (which are slightly different for neutrons and protons) are the dashed curve while the dotted curve is a plane wave calculation assuming equal neutron and proton densities. Finally, the solid curve is a full distorted wave calculation based on rel. mean field densities. 


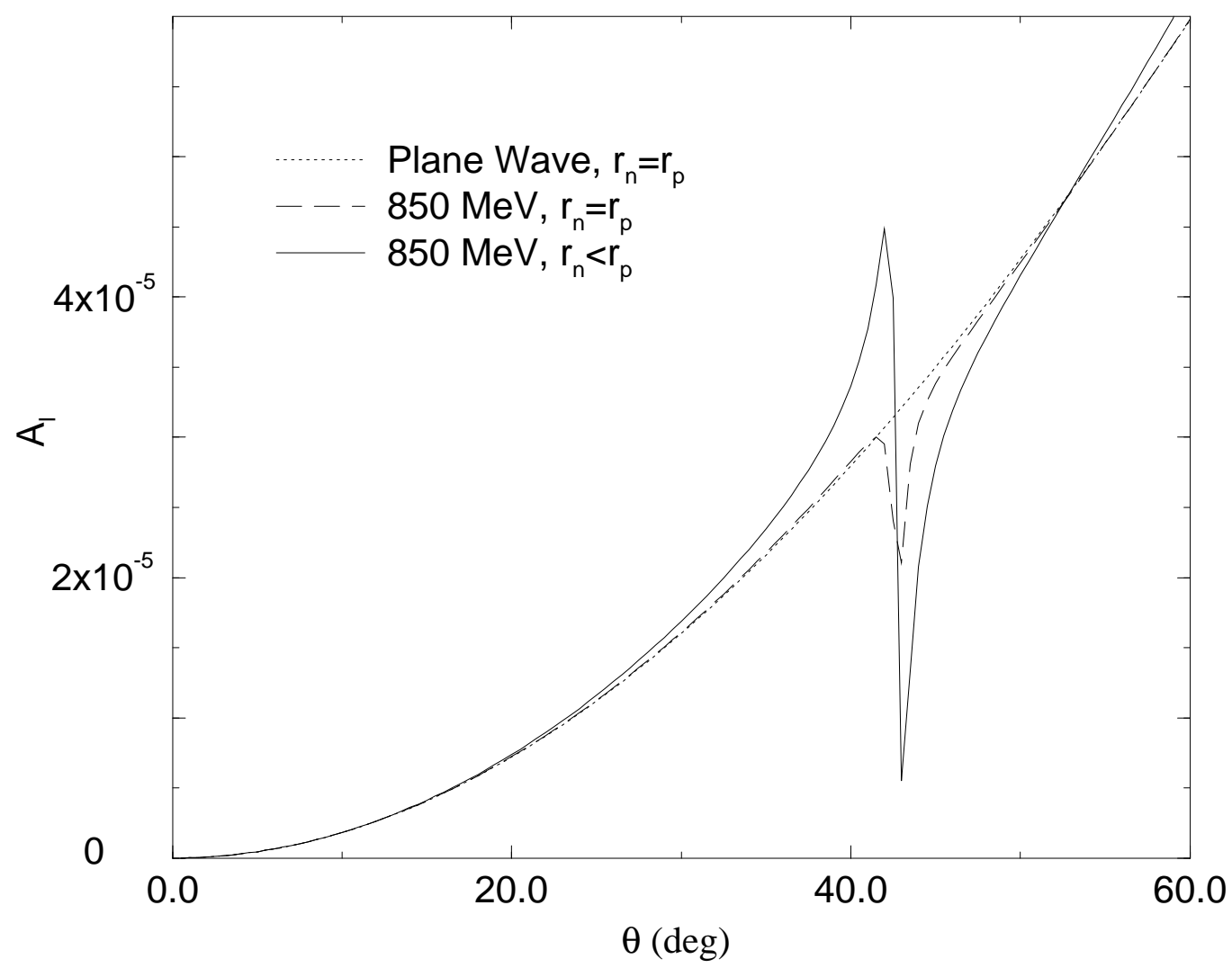

FIG. 4. Parity violating asymmetry $A_{l}$ for elastic scattering from ${ }^{4} \mathrm{He}$ at $850 \mathrm{MeV}$ vs. scattering angle $\theta$. Plane wave results using equal weak and charge densities (assumed to be a three parameter Fermi function) are the dotted curve. Distorted wave calculations with equal weak and charge densities are dashed and the solid curve includes distortions assuming the proton radius is one percent larger then the neutron radius. 


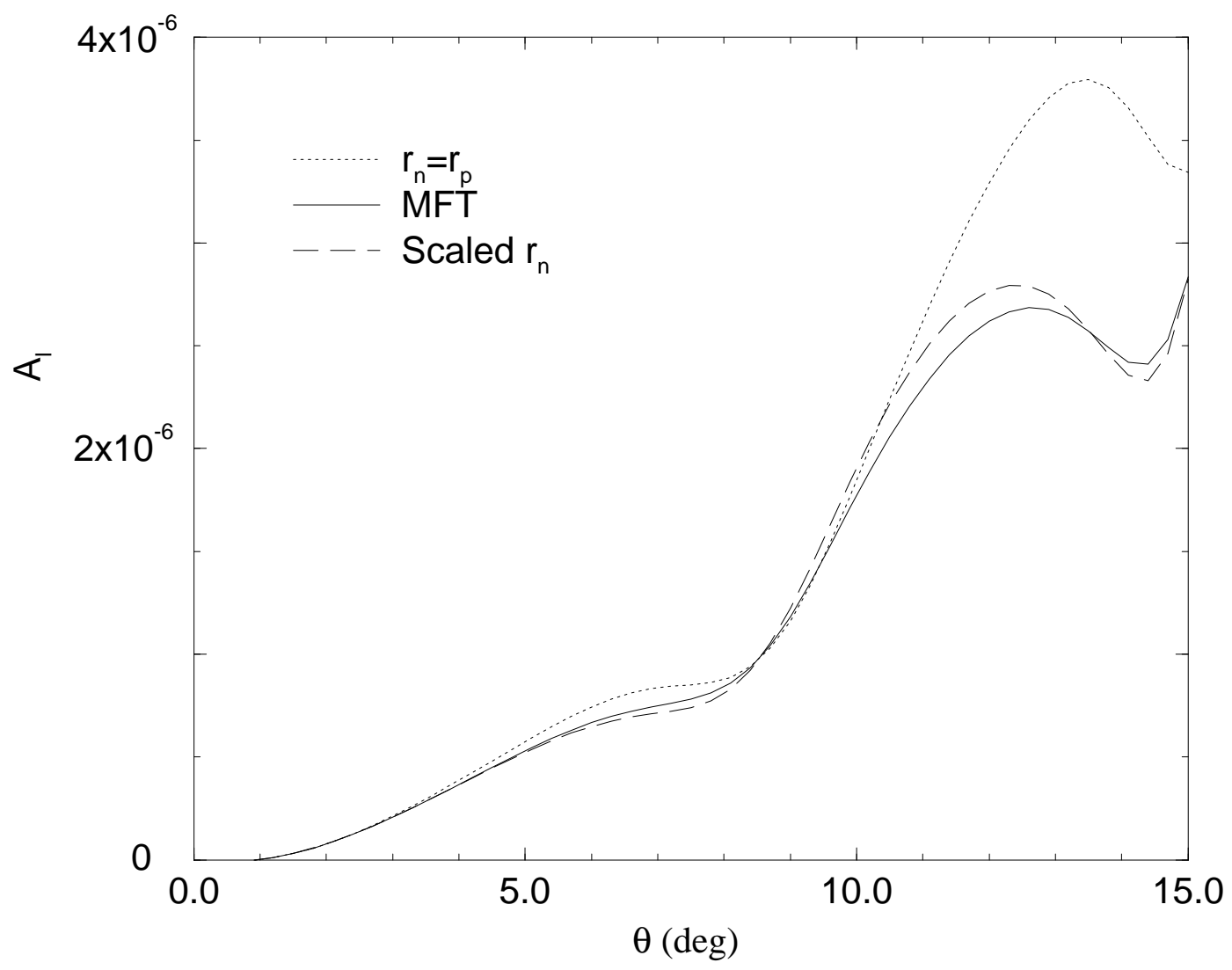

FIG. 5. Parity violating asymmetry $A_{l}$ for ${ }^{208} \mathrm{~Pb}$ at $850 \mathrm{MeV}$ vs. scattering angle $\theta$. The dotted curve uses equal weak and charge densities (assumed to be three parameter Fermi functions) while the solid curve is based on relativistic mean field densities. Finally the dashed curve assumes three parameter Fermi densities, however the weak density has been stretched (with $\lambda=.9502$ see Eq. 11) to give the same difference in radii $r_{n}-r_{p}$ as the rel. mean field densities. These densities are shown in Fig. 7. Note, all curves in this and latter figures include distortions. 


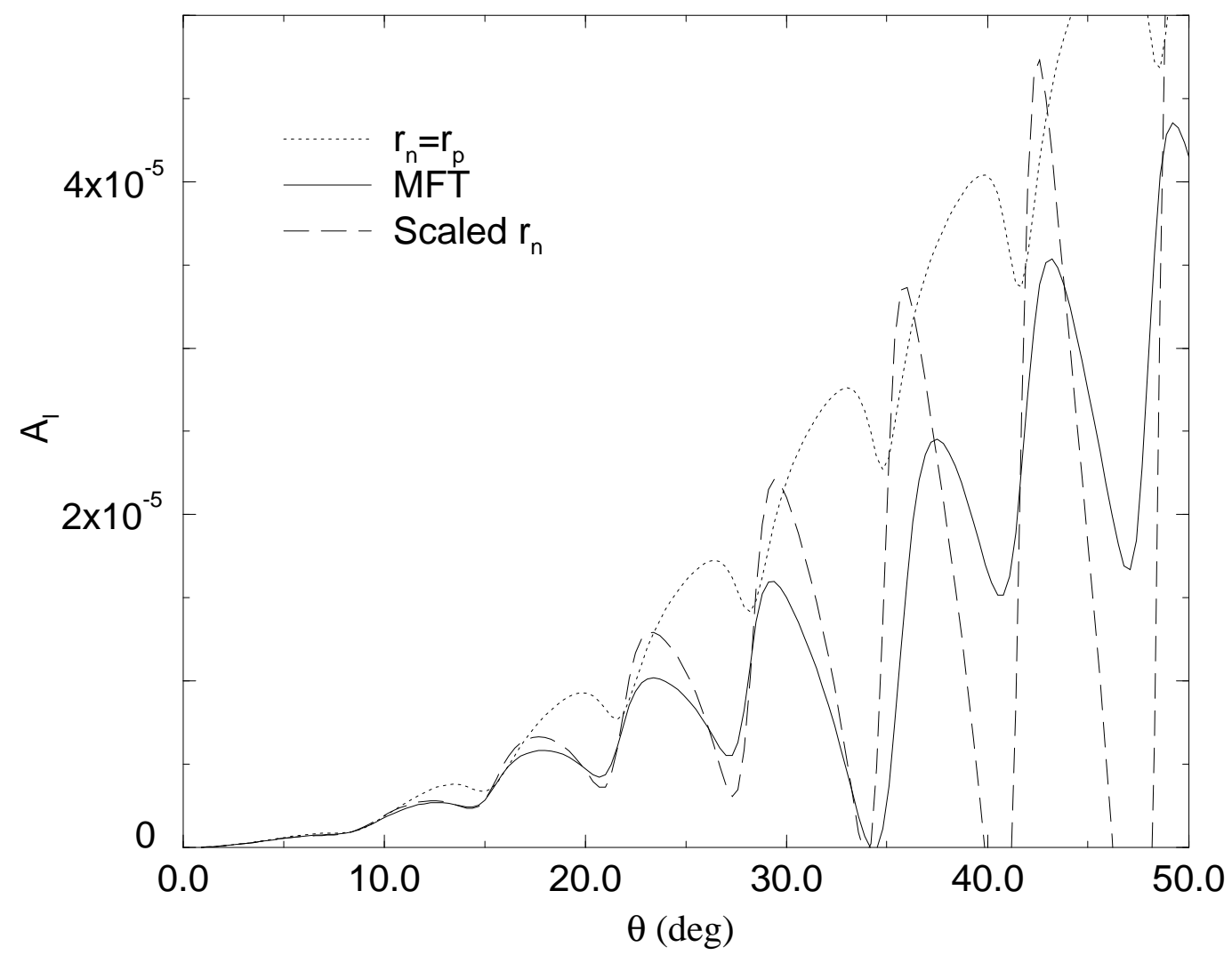

FIG. 6. As Fig. 5 except for larger scattering angles. 


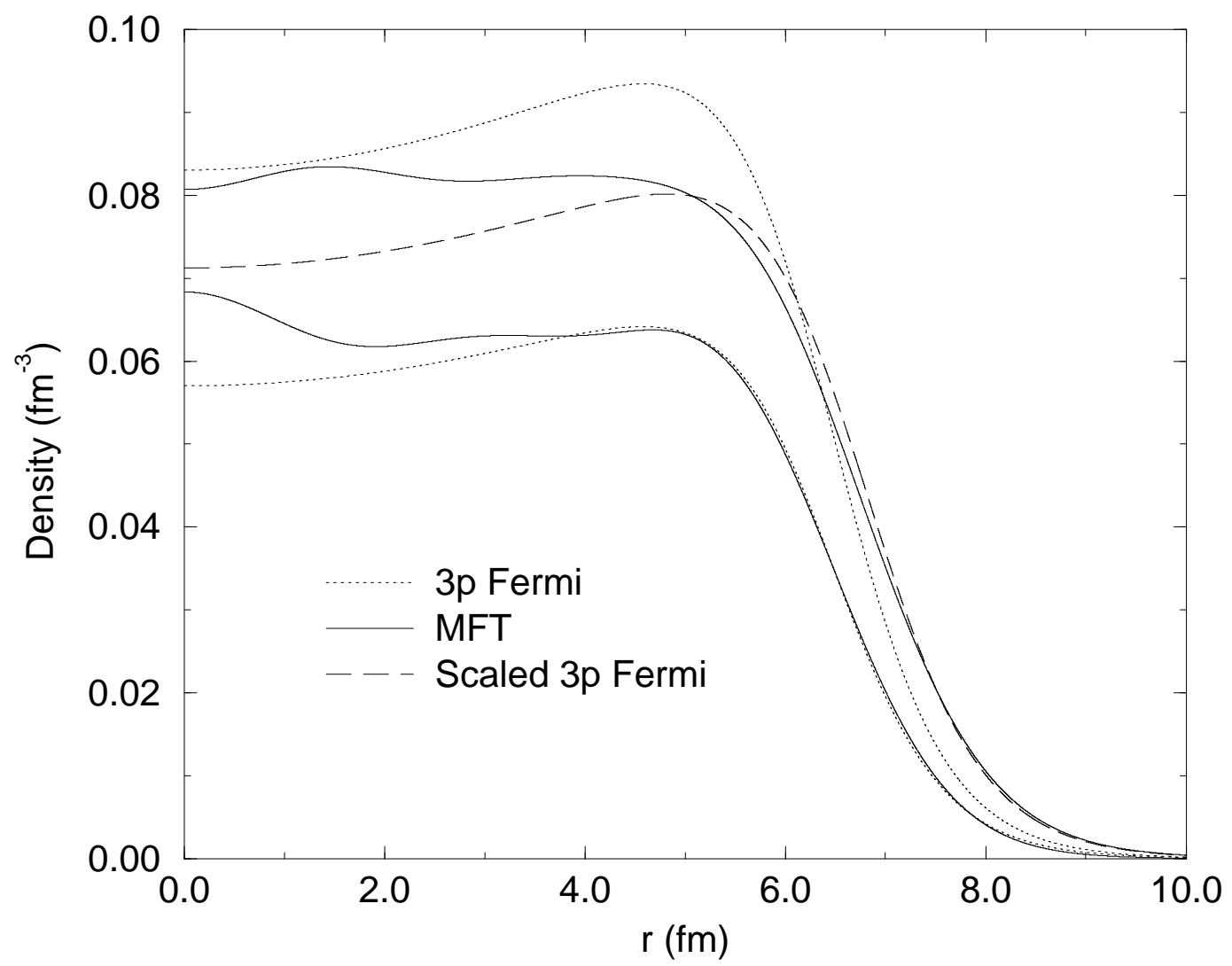

FIG. 7. Densities of ${ }^{208} \mathrm{~Pb}$ vs. radius $r$. The lower two curves are charge densities: the solid curve is the relativistic mean field result [10] while the dotted curve is a three parameter Fermi fit to elastic scattering. The upper three curves are (minus the) weak density: solid, rel. mean field, dotted, three parameter Fermi charge density normalized as in Eq. (9) while the dashed curve is this three parameter Fermi stretched by $\lambda=.9502$, see Eq. (11). 


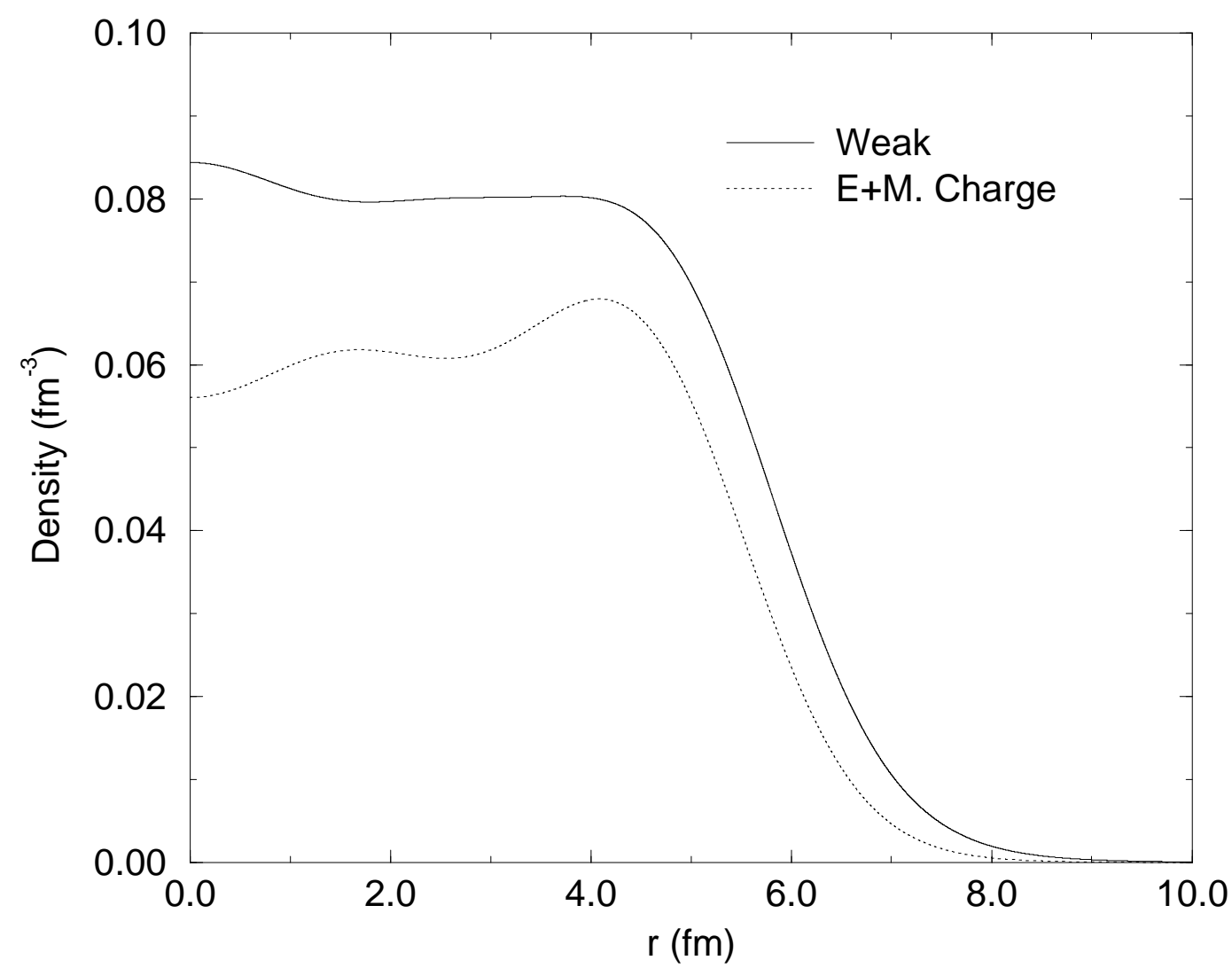

FIG. 8. Densities of ${ }^{138} \mathrm{Ba}$ vs. radius $r$ for a relativistic mean field calculation [10]. The solid curve is minus the weak density while the charge density is dotted. 


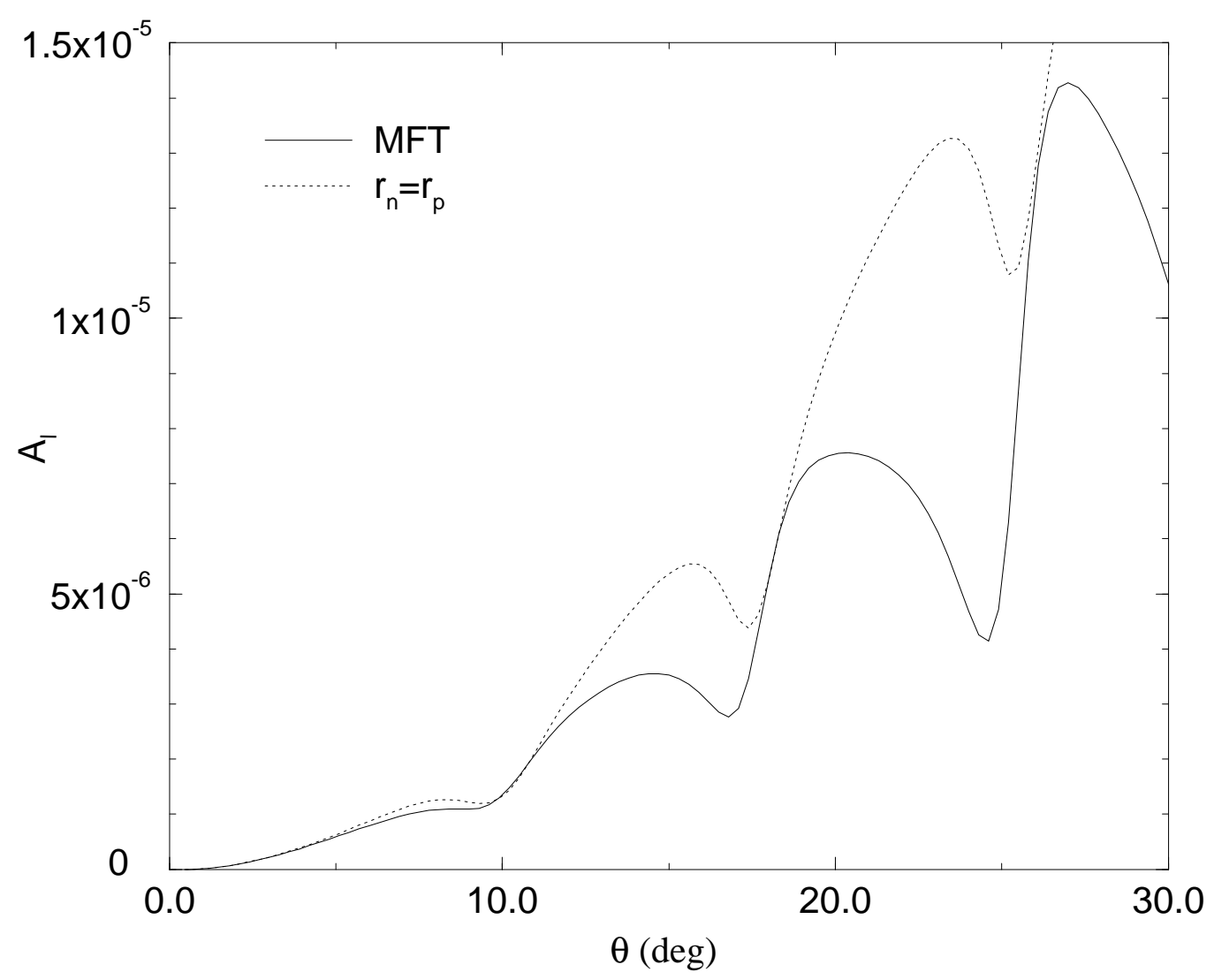

FIG. 9. Parity violating asymmetry $A_{l}$ for ${ }^{138} \mathrm{Ba}$ at $850 \mathrm{MeV}$ vs. scattering angle $\theta$. The solid curve is based on relativistic mean field densities while the dotted curve assumes equal weak and charge densities. 


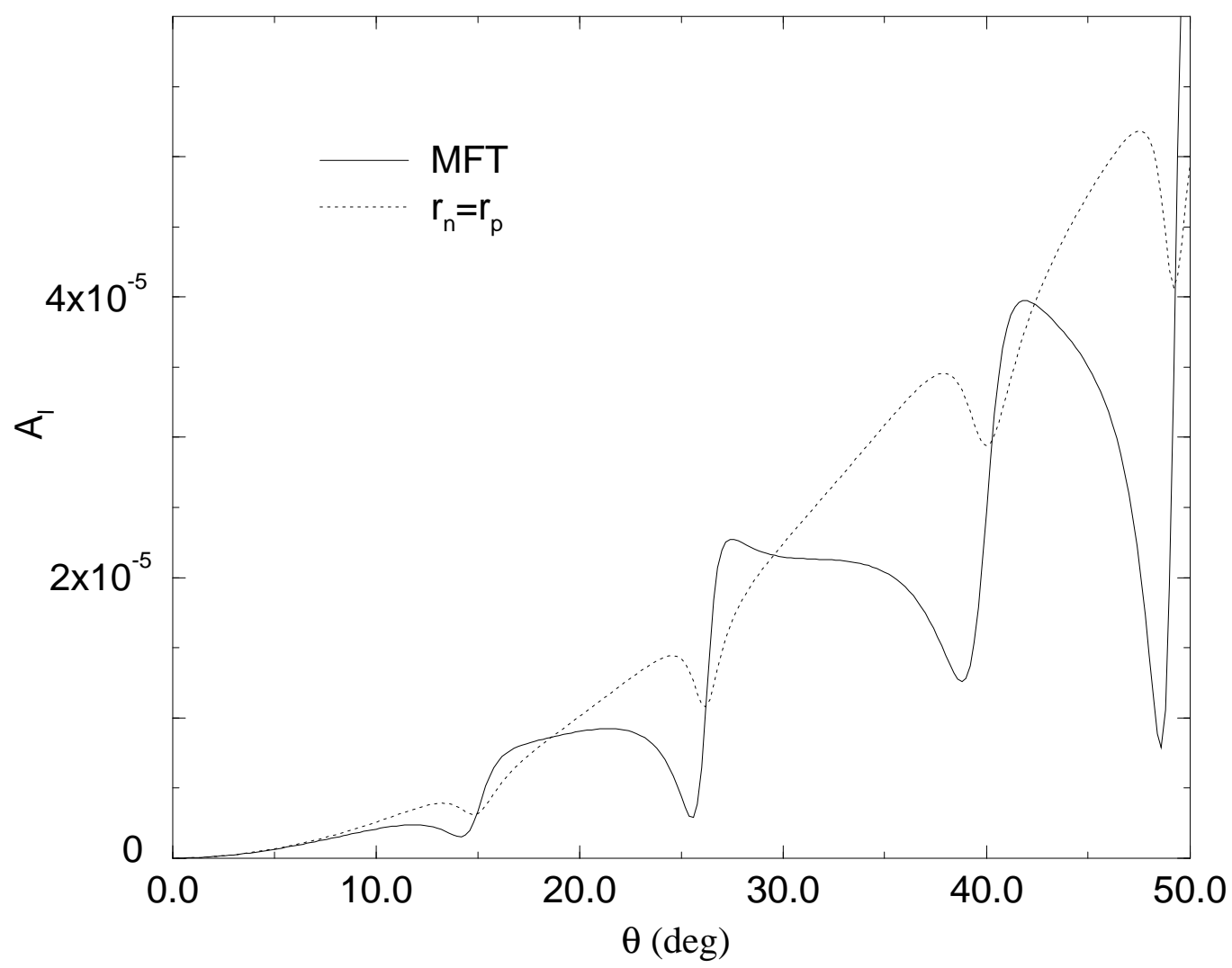

FIG. 10. Parity violating asymmetry $A_{l}$ for ${ }^{48} \mathrm{Ca}$ at $850 \mathrm{MeV}$ vs. scattering angle $\theta$. The solid curve is based on relativistic mean field densities while the dotted curve assumes equal weak and charge densities. 


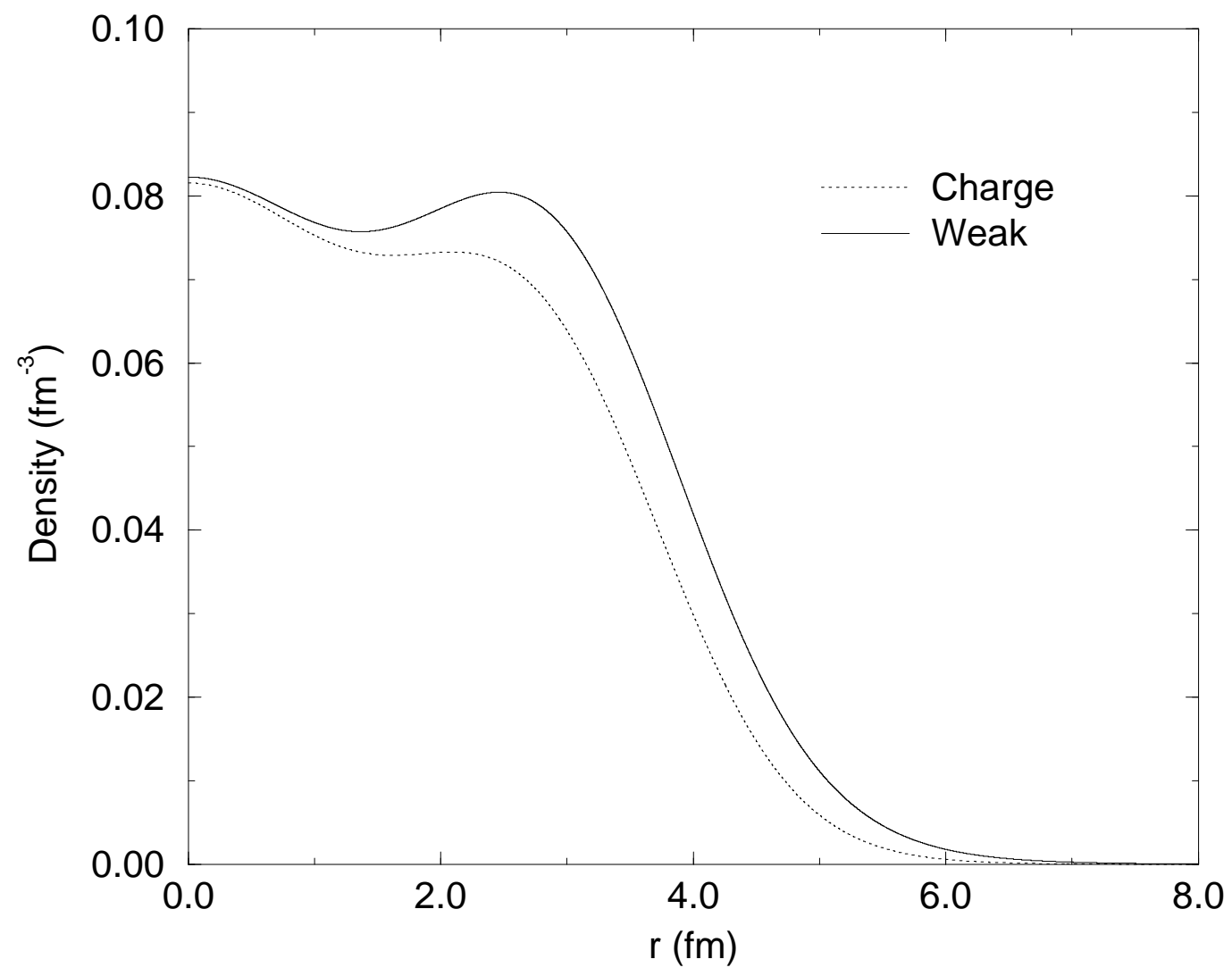

FIG. 11. Densities of ${ }^{48} \mathrm{Ca}$ vs. radius $r$ for a relativistic mean field calculation [10]. The solid curve is minus the weak density while the charge density is dotted. 


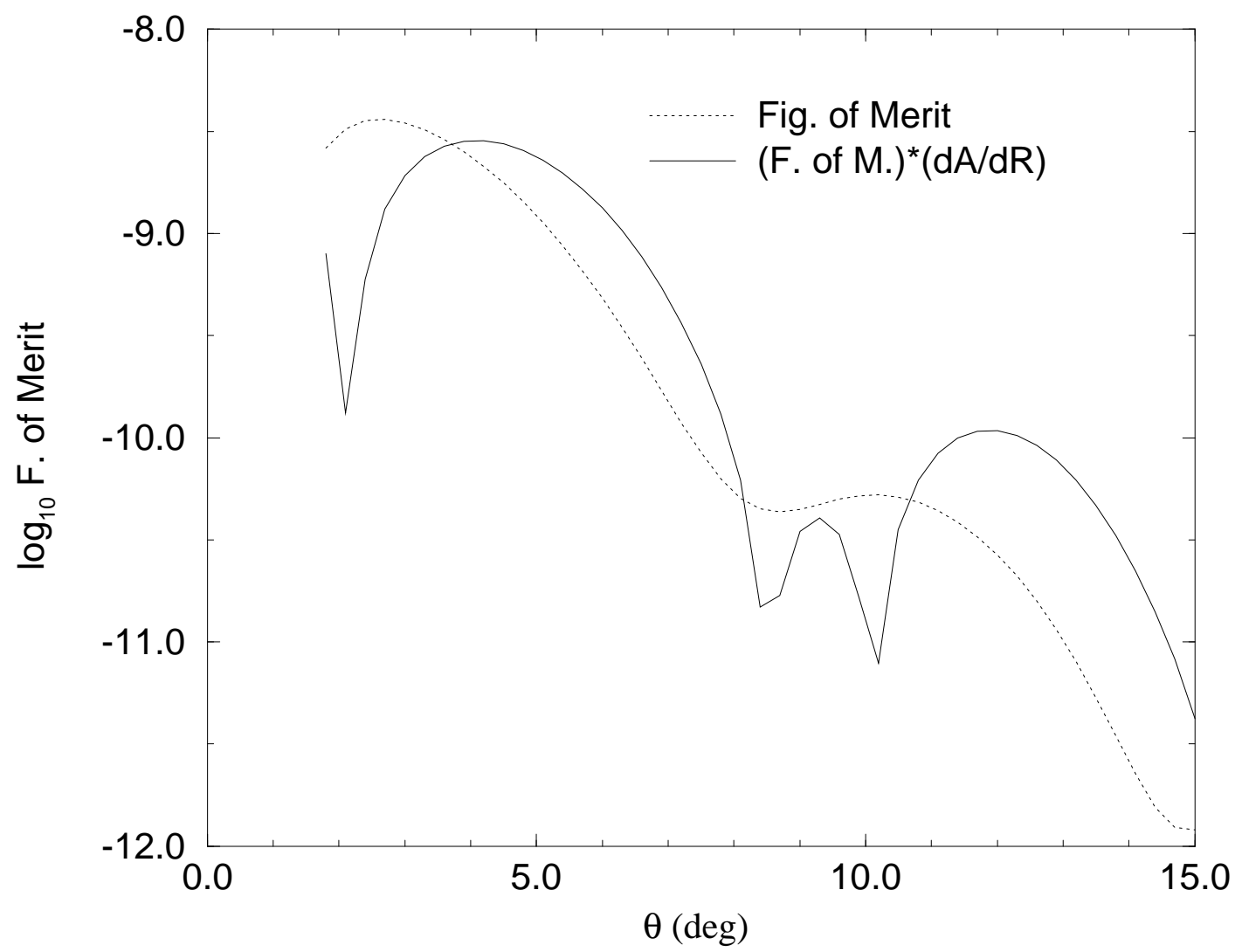

FIG. 12. Figure of merit, dotted curve, (differential cross section times asymmetry $A_{l}$ squared) $\log _{10}$ in $\mathrm{mb} / \mathrm{Sr}$ vs. scattering angle $\theta$ for ${ }^{208} \mathrm{~Pb}$ at $850 \mathrm{MeV}$. The solid curve is the figure of merit multiplied by the logarithmic derivative of the asymmetry with respect to the neutron radius (see Fig. 13). 


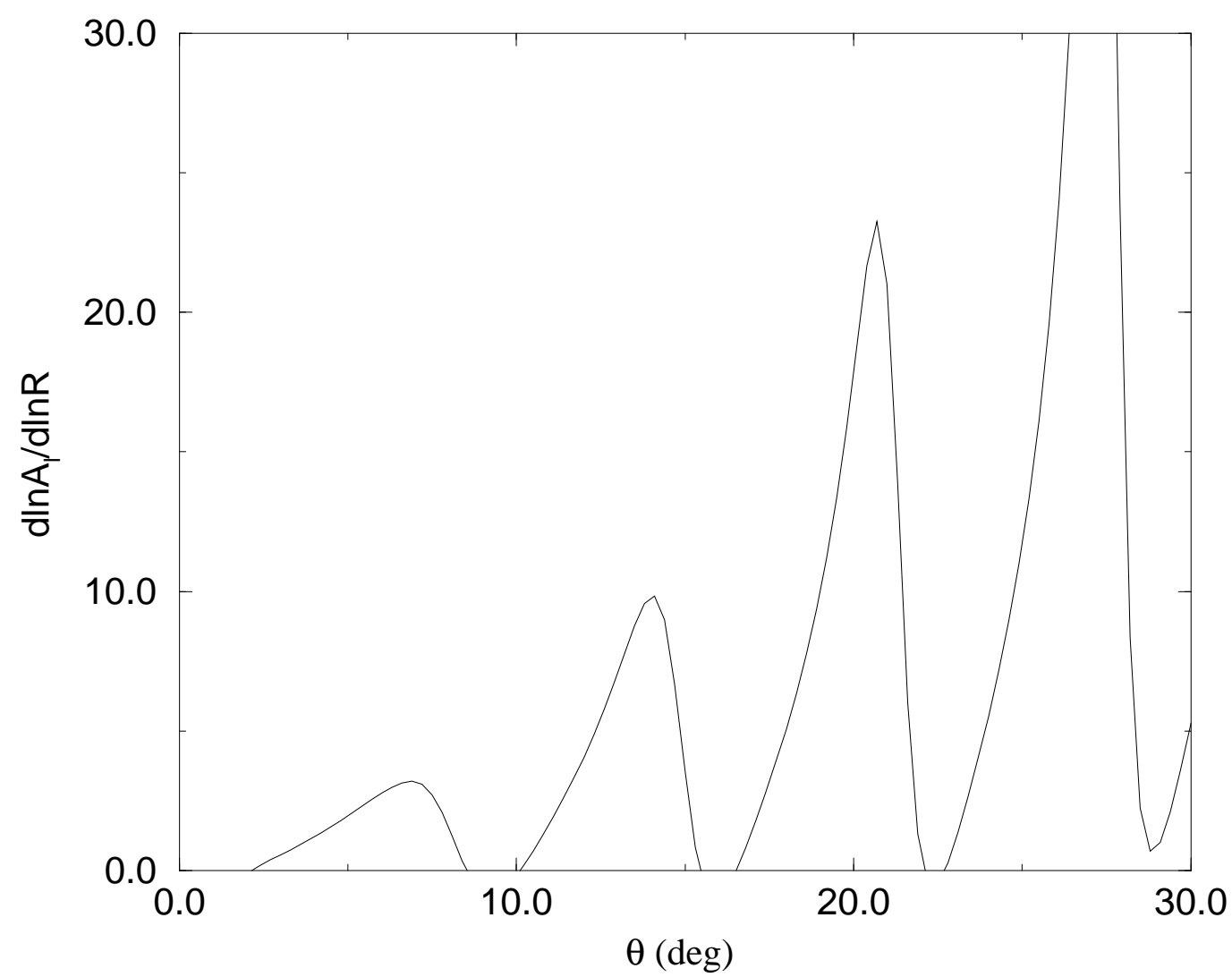

FIG. 13. Logarithmic derivative of the asymmetry $A_{l}$ with respect to the neutron radius for ${ }^{208} \mathrm{~Pb}$ at $850 \mathrm{MeV}$ vs. scattering angle $\theta$. 


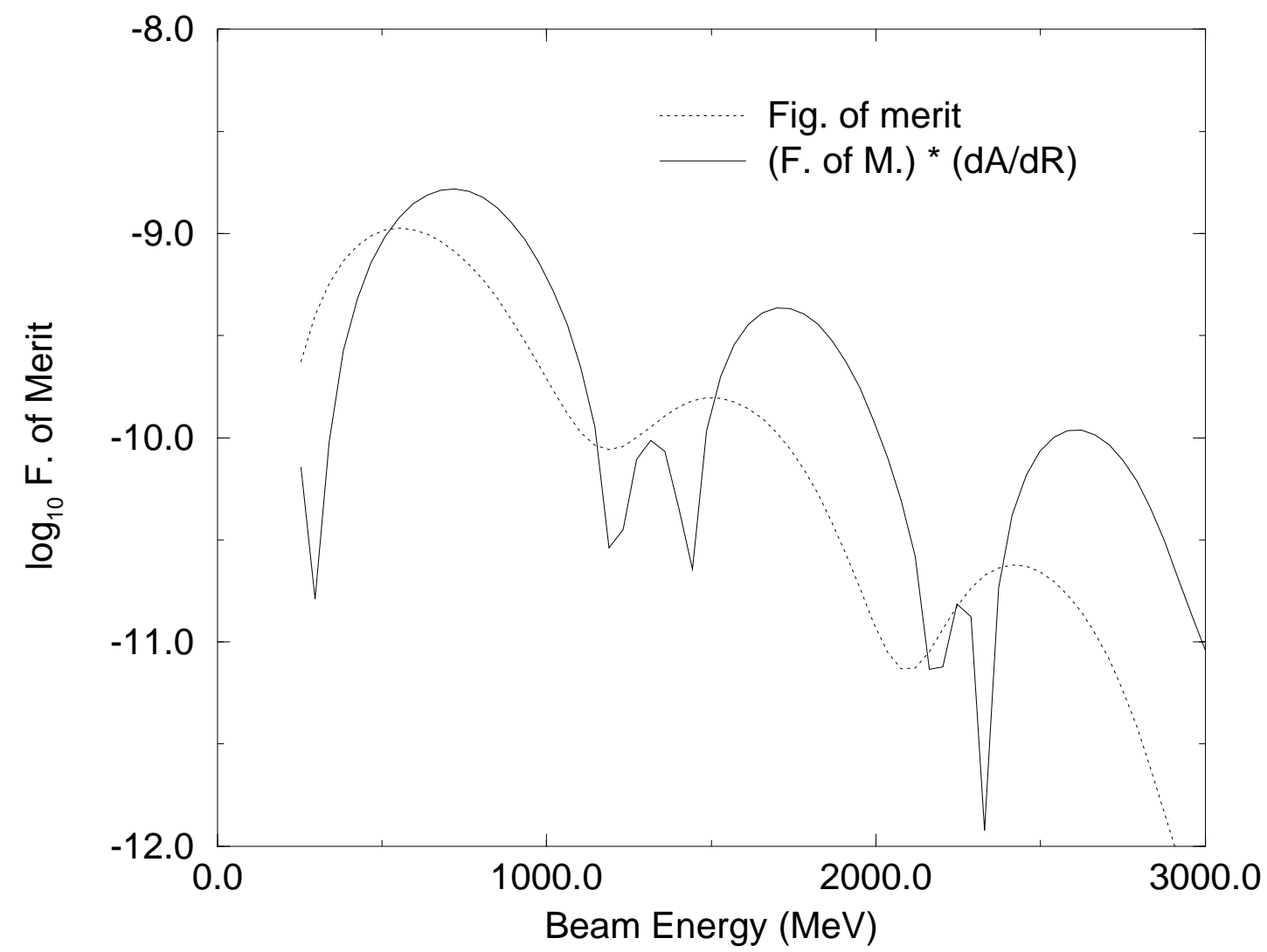

FIG. 14. Figure of merit, dotted curve, vs. beam energy for a fixed laboratory scattering angle of six degrees for ${ }^{208} \mathrm{~Pb}$. The solid curve is the product of the figure of merit times the logarithmic derivative of the asymmetry with respect to the neutron radius. Note these curves are approximate. They are based on distortions calculated at $850 \mathrm{MeV}$ and assumed independent of energy. 